\title{
Use of a nonpledgeted suture technique is safe and efficient for aortic valve replacement
}

\author{
Damien J. LaPar, MD, Gorav Ailawadi, MD, Castigliano M. Bhamidipati, DO, Mansher Singh, MBBS, \\ David Dare, BA, John A. Kern, MD, and Irving L. Kron, MD
}

\begin{abstract}
Objective: The use of pledgeted sutures to secure the prosthetic valve to the annulus during aortic valve replacement is thought to decrease the incidence of paravalvular leak. We hypothesized that use of nonpledgeted sutures in aortic valve replacement would provide equivalent outcomes to those of a pledgeted suture technique.
\end{abstract}

\begin{abstract}
Methods: Between January 1995 and April 2009, a total of 802 patients (511 nonpledgeted, 291 pledgeted) underwent isolated aortic valve replacement, including 671 patients who underwent primary, isolated aortic valve replacement (412 nonpledgeted, 259 pledgeted). Preoperative risk, intraoperative findings, and postoperative complications, including operative mortality, were evaluated.
\end{abstract}

\begin{abstract}
Results: Operative mortalities in isolated AVR operations were similar at $2.5 \%$ and $3.1 \%(P>.66)$ for nonpledgeted and pledgeted groups, respectively. Paravalvular leak rates after aortic valve replacement were equivalent in nonpledgeted and pledgeted groups $(0.8 \%$ vs $1.4 \%$, respectively, $P=.47)$. Reoperation for paravalvular leak was rare in both groups. Importantly, the nonpledgeted technique incurred significantly shorter aortic crossclamp time $(58.1 \pm 0.3$ minutes vs $61.6 \pm 0.4$ minutes, $P<.001)$ and cardiopulmonary bypass time $(87.5 \pm 0.8$ minutes vs $90.3 \pm 0.8$ minutes, $P=.02$ ) than did the pledgeted technique.
\end{abstract}

Conclusions: A nonpledgeted suture technique offers an equivalent alternative to the traditional use of pledgets during aortic valve replacement, with no increase in paravalvular leak rate. This nonpledgeted suture technique provides a time efficient and safe approach to aortic valve replacement operations. (J Thorac Cardiovasc Surg 2011;141:388-93)

Aortic valve disease is a common condition with advancing age, and aortic stenosis is the most common valve condition for which aortic valve replacement (AVR) is performed within the United States. According to the Society for Thoracic Surgeons (STS), approximately 12,000 to 18,000 isolated AVR operations are performed annually, with operative mortalities approaching $3 \% .{ }^{1}$ Current demographic trends indicate a significant increase in the US geriatric population, resulting from the rising "baby boomer" population coupled with the increase in human life span. Clinically significant calcific aortic stenosis requiring AVR is common within this population, occurring in approximately $20 \%$ of patients. ${ }^{2}$ Consequently, evaluation of current approaches to operative management of aortic valve disease is justified.

Major paravalvular leak after AVR is a serious complication and a significant risk, and the classic surgical approach to open AVR attempts to avoid leaks through the use of

From the Division of Thoracic and Cardiovascular Surgery, Department of Surgery, University of Virginia, Charlottesville, Va.

Supported by National Institutes of Health National Heart, Lung and Blood Institute T32 Training Grant HL007849 (to D.J.L., C.M.B.) and Thoracic Surgery Foundation for Research and Education Research Grant (to G.A.).

Disclosures: Authors have nothing to disclose with regard to commercial support.

Received for publication Dec 16, 2009; revisions received Feb 16, 2010; accepted for publication April 10, 2010; available ahead of print May 21, 2010.

Address for reprints: Gorav Ailawadi, MD, PO Box 800679, Charlottesville, VA 22908-0679 (E-mail: gorav@virginia.edu).

$0022-5223 / \$ 36.00$

Copyright (c) 2011 by The American Association for Thoracic Surgery doi:10.1016/j.jtcvs.2010.04.011 pledgeted, nonabsorbable sutures to secure the prosthesis to the aortic annulus. Clinically significant paravalvular leak necessitating reoperation has been reported to occur in fewer than $2 \%$ of cases. ${ }^{3}$ Within the United States, the use of pledgets during AVR is common. Pledgeted sutures to the secure the valve prosthesis to the valve annulus during mitral, aortic, and double mechanical valve replacements have been shown to have a protective effect against postoperative paravalvular leak in comparisons with several different suture techniques. ${ }^{4}$ To our knowledge, however, the effect of a uniform, nonpledgeted suture technique during AVR has not been previously well established.

At our institution, we have adopted the routine use of nonpledgeted sutures during AVR because we believe this approach to be efficient and yet carry minimal risk of major paravalvular leak. Accordingly, in this study, we sought to evaluate the use of a nonpledgeted suture technique during AVR. We hypothesized that a nonpledgeted AVR technique would be equivalent to a pledgeted technique with respect to operative mortality, major paravalvular leak rate, and need for subsequent aortic valve reoperation.

\section{MATERIALS AND METHODS Patients}

This study was approved by the human investigation committee of the University of Virginia Health System (HSR 14064). All AVR operations at our institution were entered prospectively into the STS National Database. We retrospectively reviewed all patients undergoing isolated AVR from January 1995 to April 2009. Primary AVRs were defined as those occurring in the 


\section{Abbreviations and Acronyms}

$\mathrm{AVR}=$ aortic valve replacement

absence of previous sternotomy or cardiac operation. Patients were stratified according to suture technique into nonpledgeted and pledgeted AVR groups. In subgroup analysis, we further studied patients undergoing primary, isolated AVR to eliminate the confounding effects of reoperation on outcomes.

Patient characteristics, risk factors, operative features, and postoperative outcomes were evaluated. We used known STS definitions to describe all preoperative and operative variables as well as postoperative outcomes. Operative mortality included patient deaths occurring before hospital discharge or within 30 days after the operation. Incidence of major paravalvular leak was evaluated immediately after AVR with transesophageal echocardiography, and transthoracic echocardiography was used to detect major paravalvular leak during postoperative follow-up.

\section{Operative Technique}

Standard AVR procedures were used in all cases. A complete median sternotomy was performed. Intraoperative transesophageal echocardiography was routinely used to assess the diseased aortic valve before sternotomy. After cardiopulmonary bypass was established and the aortic crossclamp placed, cardioplegia was administered into the aortic root through a retrograde coronary sinus catheter. A transverse aortotomy was made 2 to $3 \mathrm{~cm}$ distal to the origin of the right coronary artery, the diseased aortic valve was visualized, and the valve leaflets were carefully excised, including débridement of all calcific deposits within the aortic annulus. The valve prosthesis was sized, selected, and sewn to the aortic annulus with 12 to 18 sutures. In the pledgeted group, 2-0 Ti-Cron sutures (Covidien, Mansfield, Mass) with premade pledgets were placed in a horizontal mattress fashion, placing the sutures from the ventricular side of the annulus to the aortic side of the annulus. In the nonpledgeted group, 2-0 Ti-Cron sutures without pledgets were placed in a similar fashion. With both techniques, the sutures were then passed through the sewing ring of the prosthetic valve, and the valve was secured in a supraannular position. No continuous or simple, interrupted sutures were used. The aortotomy was closed with running nonabsorbable polypropylene suture in 2 layers. Deairing was performed, and the aortic crossclamp was then removed. During the deairing process, transesophageal echocardiography was used to verify adequate deairing and to assess the newly implanted prosthesis for competence and the presence of paravalvular leak. The patient was then weaned from cardiopulmonary bypass, pacing wires and drainage tubes were placed, and the wound was closed in the standard surgical fashion.

\section{Statistical Analysis}

Our primary outcomes of interest were operative mortality, major paravalvular leak rate, and need for aortic valve reoperation for major paravalvular leak among all patients undergoing nonpledgeted versus pledgeted AVR. All group comparisons were unpaired. Bivariate comparisons with either Pearson $\chi^{2}$ or Fisher's Exact tests were used for all categoric variables. Analysis of variance was used for all continuous variables.

Categoric variable comparisons are expressed as percentages of each group of origin. Continuous variables are reported as mean \pm SEM. Reported $P$ values are 2-tailed. Data manipulation and analysis were performed with SPSS software (version 17; SPSS Inc, an IBM Company, Chicago, Ill).

\section{RESULTS}

\section{Comparison of Patient Characteristics and Operative Features}

A total of 802 patients (511 nonpledgeted vs 291 pledgeted) underwent isolated AVR operations from January
1995 to April 2009 (Table 1). The average patient age was higher among patients undergoing nonpledgeted AVR $(67.0 \pm 0.7$ years) than those undergoing pledgeted AVR (62.7 \pm 1.5 years, $P=.01$ ). Previous coronary artery bypass grafting operations had been performed in $13.3 \%$ and $4.5 \%$ $(P<.001)$ of nonpledgeted and pledgeted patient groups, respectively. The prevalences of previous valve operations were similar. Infective endocarditis incidences were similar between nonpledgeted and pledgeted AVR groups. Patients undergoing isolated, pledgeted AVR had statistically lower preoperative ejection fractions $(51.8 \%)$ than did those undergoing isolated, nonpledgeted AVR $(53.6 \%, P=.03)$. Aortic crossclamp time $(58.1 \pm 0.3$ minutes vs $61.6 \pm 0.4$ minutes, $P<.001$ ) and cardiopulmonary bypass time ( $87.5 \pm 0.8$ minutes vs $90.3 \pm 0.8$ minutes, $P=.02$ ) were shorter in the nonpledgeted AVR group than in the pledgeted AVR group.

Similar trends in patient demographic characteristics, risk factors, and operative features were observed among patients undergoing primary, isolated AVR (Table 2). A total of 671 patients (412 nonpledgeted vs 259 pledgeted) underwent primary, isolated AVR. The nonpledgeted AVR group was older $(P=.004)$, with higher rates of preoperative cerebrovascular disease $(P=.04)$, dyslipidemia $(P<.001)$, and hypertension $(P<.001)$. Intraoperatively, the nonpledgeted group had shorter aortic crossclamp time $(57.8 \pm 0.3$ vs $61.6 \pm 0.5$ minutes, $P<.001)$ and cardiopulmonary bypass time $(84.7 \pm 0.6$ vs $89.5 \pm 0.8$ minutes, $P<.001)$ than did the pledgeted group. Urgent and emergency operations were more common among patients undergoing primary, isolated nonpledgeted AVR.

\section{Comparison of Early and Late Outcomes}

An analysis of outcomes of all patients undergoing isolated AVR revealed no significant differences between nonpledgeted and pledgeted groups (Table 3). Operative mortalities were similarly low in both AVR operative groups. Paravalvular leaks and the need for reoperation were similarly rare. Mean patient follow-up was $82.0 \pm 1.9$ months. Eight cases of major paravalvular leak occurred at $51.3 \pm 16.7$ months after either nonpledgeted or pledgeted AVR. Patient characteristics and operative features for patients incurring major paravalvular leaks appear in Table 4. Among patients undergoing primary, isolated AVR (Table 5), operative mortalities were similar $(1.9 \%$ vs $2.3 \%, P=.79)$ in the nonpledgeted and pledgeted AVR groups. Moreover, paravalvular leaks after primary, isolated AVR $(P=.38)$ and the need for reoperation for paravalvular leak $(P=.06)$ were both uncommon and comparable between groups. Total hospital of stay was longer among patients undergoing primary, isolated pledgeted AVR $(P=.02)$. None of the patients with a preoperative history of endocarditis incurred a major paravalvular leak after AVR. All other measured postoperative outcomes were similar regardless of nonpledgeted or pledgeted AVR technique. 
TABLE 1. Preoperative and operative variables for patients undergoing isolated aortic valve replacement with a nonpledgeted versus pledgeted suture technique $(n=802)$

\begin{tabular}{|c|c|c|c|}
\hline Variable & Nonpledgeted $(n=511)$ & Pledgeted $(n=291)$ & $P$ value \\
\hline \multicolumn{4}{|l|}{ Preoperative } \\
\hline Age $(y$, mean \pm SEM $)$ & $67.0 \pm 0.7$ & $62.7 \pm 1.5$ & .01 \\
\hline Sex (no. female) & $200(39.1 \%)$ & $122(41.9 \%)$ & .45 \\
\hline Hypertension (no.) & $363(71.0 \%)$ & $146(50.2 \%)$ & $>.001$ \\
\hline Peripheral arterial disease (no.) & $49(9.6 \%)$ & $19(6.5 \%)$ & .15 \\
\hline Stroke (no.) & $39(7.6 \%)$ & $10(3.4 \%)$ & .02 \\
\hline Diabetes mellitus (no.) & $104(20.4 \%)$ & $47(16.2 \%)$ & .16 \\
\hline Dyslipidemia (no.) & $310(60.7 \%)$ & $83(28.5 \%)$ & $>.001$ \\
\hline Atrial fibrillation (no.) & $93(18.2 \%)$ & $32(11.0 \%)$ & .01 \\
\hline Heart block (no.) & $11(2.2 \%)$ & $8(2.7 \%)$ & .63 \\
\hline Ventricular tachycardia (no.) & $1(0.2 \%)$ & $2(0.7 \%)$ & .30 \\
\hline \multicolumn{4}{|l|}{ New York Heart Association functional class (no.) } \\
\hline I & $235(46.0 \%)$ & $146(49.0 \%)$ & .43 \\
\hline II & $93(18.2 \%)$ & $62(20.8 \%)$ & .40 \\
\hline III & $119(23.3 \%)$ & $58(19.5 \%)$ & .22 \\
\hline IV & $64(12.5 \%)$ & $32(10.7 \%)$ & .50 \\
\hline Heart failure (no.) & $191(37.4 \%)$ & $109(37.5 \%)$ & $>.99$ \\
\hline Infective endocarditis (no.) & $34(6.7 \%)$ & $17(5.8 \%)$ & .76 \\
\hline Renal failure (no.) & $42(8.2 \%)$ & $24(8.2 \%)$ & $>.99$ \\
\hline Hemodialysis (no.) & $10(2.0 \%)$ & $7(2.4 \%)$ & .80 \\
\hline Ejection fraction $(\%$, mean \pm SEM $)$ & $53.6 \pm 0.5$ & $51.8 \pm 0.6$ & .03 \\
\hline Previous coronary artery bypass grafting (no.) & $68(13.3 \%)$ & $13(4.5 \%)$ & $>.001$ \\
\hline Previous valve operation (no.) & $42(8.2 \%)$ & $20(6.9 \%)$ & .58 \\
\hline \multicolumn{4}{|l|}{ Aortic insufficiency (no.) } \\
\hline Moderate & $57(11.2 \%)$ & $52(17.9 \%)$ & .01 \\
\hline Severe & $92(18.0 \%)$ & $48(16.5 \%)$ & .63 \\
\hline Aortic stenosis (no.) & $427(83.6 \%)$ & $216(74.2 \%)$ & .004 \\
\hline \multicolumn{4}{|l|}{ Operative } \\
\hline \multicolumn{4}{|l|}{ Urgency (no.) } \\
\hline Elective & $386(75.5 \%)$ & $232(79.7 \%)$ & .19 \\
\hline Urgent & $119(23.3 \%)$ & $58(19.9 \%)$ & .29 \\
\hline Emergency & $6(1.2 \%)$ & $1(0.3 \%)$ & .43 \\
\hline \multicolumn{4}{|l|}{ Prosthesis type (no.) } \\
\hline Bioprosthesis & $431(84.3 \%)$ & $160(55.0 \%)$ & $>.001$ \\
\hline Mechanical prosthesis & $77(15.1 \%)$ & $122(41.9 \%)$ & $>.001$ \\
\hline Homograft & $3(0.6 \%)$ & $9(3.1 \%)$ & .01 \\
\hline Aortic crossclamp time (min, mean \pm SEM) & $58.1 \pm 0.3$ & $61.6 \pm 0.4$ & $>.001$ \\
\hline Cardiopulmonary bypass time (min, mean \pm SEM) & $87.5 \pm 0.8$ & $90.3 \pm 0.8$ & .02 \\
\hline
\end{tabular}

\section{DISCUSSION}

In this study, we have corroborated current evidence to suggest improved outcomes among open AVR operations, and we have highlighted a time efficient and clinically equivalent approach to AVR with a nonpledgeted suture technique to secure the aortic valve prosthesis to the aortic annulus. Our study groups were well matched with respect to patient demographic characteristics and preoperative risk factors. Trends in postoperative outcomes between study groups were unchanged by the inclusion of reoperative isolated AVR procedures. Most importantly, operative mortality, major paravalvular leak rate, and need for reoperation for major paravalvular leak were equivalent in nonpledgeted and pledgeted AVR operations. Further, a nonpledgeted technique allowed statistically shorter aortic crossclamp and total cardiopulmonary bypass times. We believe that this study provides important contributions to the examination of contemporary outcomes of open AVR operations.

Major paravalvular leak remains a serious complication and primary concern after AVR. Small, clinically benign, paravalvular leaks are common in the early postoperative setting and are well tolerated. ${ }^{5}$ Larger, major paravalvular leaks, however, occur after $1 \%$ to $5 \%$ of AVR procedures and have greater clinical relevance. Such leaks have been shown to be associated with heavy annular calcification. ${ }^{5-7}$ Although significant paravalvular leaks initially may be managed medically, reoperative AVR is frequently required for definitive management. Reoperation in turn 
TABLE 2. Preoperative and operative variables for all patients undergoing primary, isolated AVR with a nonpledgeted versus pledgeted suture technique $(\mathbf{n}=671)$

\begin{tabular}{|c|c|c|c|}
\hline Variable & Nonpledgeted $(n=412)$ & Pledgeted $(n=259)$ & $P$ value \\
\hline \multicolumn{4}{|l|}{ Preoperative } \\
\hline Age $(y$, mean \pm SEM $)$ & $67.2 \pm 0.7$ & $62.3 \pm 1.5$ & .994 \\
\hline Sex (no. female) & $174(42.2 \%)$ & $115(44.4 \%)$ & .63 \\
\hline Hypertension (no.) & $279(67.7 \%)$ & $126(48.6 \%)$ & $>.001$ \\
\hline Peripheral arterial disease (no.) & $31(7.5 \%)$ & $15(5.8 \%)$ & .44 \\
\hline Stroke (no.) & $28(6.8 \%)$ & $9(3.5 \%)$ & .08 \\
\hline Diabetes mellitus (no.) & $76(18.4 \%)$ & $38(14.7 \%)$ & .25 \\
\hline Dyslipidemia (no.) & $232(56.3 \%)$ & $66(25.5 \%)$ & $>.001$ \\
\hline Atrial fibrillation (no.) & $70(17.0 \%)$ & $30(11.6 \%)$ & .06 \\
\hline Heart block (no.) & $9(2.2 \%)$ & $6(2.3 \%)$ & $>.99$ \\
\hline Ventricular tachycardia (no.) & $1(0.2 \%)$ & $1(0.4 \%)$ & $>.99$ \\
\hline \multicolumn{4}{|l|}{ New York Heart Association functional class (no.) } \\
\hline I & $213(51.7 \%)$ & $123(47.5 \%)$ & .30 \\
\hline II & $87(21.1 \%)$ & $58(22.4 \%)$ & .70 \\
\hline III & $79(19.2 \%)$ & $55(21.2 \%)$ & .55 \\
\hline IV & $33(8.0 \%)$ & $23(8.9 \%)$ & .77 \\
\hline Heart failure (no.) & $155(37.6 \%)$ & $97(37.5 \%)$ & $>.99$ \\
\hline Infective endocarditis (no.) & $18(4.4 \%)$ & $10(3.9 \%)$ & .84 \\
\hline Renal failure (no.) & $33(8.0 \%)$ & $21(8.1 \%)$ & $>.99$ \\
\hline Hemodialysis (no.) & $6(1.5 \%)$ & $6(2.3 \%)$ & .55 \\
\hline Ejection fraction $(\%$, mean \pm SEM $)$ & $54.1 \pm 0.6$ & $52.1 \pm 0.7$ & .02 \\
\hline \multicolumn{4}{|l|}{ Aortic insufficiency (no.) } \\
\hline Moderate & $50(12.1 \%)$ & $45(17.4 \%)$ & .04 \\
\hline Severe & $73(17.7 \%)$ & $44(17.0 \%)$ & .84 \\
\hline Aortic stenosis (no.) & $349(84.7 \%)$ & $200(77.2 \%)$ & .02 \\
\hline \multicolumn{4}{|l|}{ Operative } \\
\hline \multicolumn{4}{|l|}{ Urgency $(\%)$} \\
\hline Elective & $311(75.5 \%)$ & $211(81.5 \%)$ & .07 \\
\hline Urgent & $96(23.3 \%)$ & $47(18.1 \%)$ & .12 \\
\hline Emergency & $5(1.2 \%)$ & $1(0.4 \%)$ & .41 \\
\hline \multicolumn{4}{|l|}{ Prosthesis type (no.) } \\
\hline Bioprosthesis & $354(85.9 \%)$ & $143(55.2 \%)$ & $>.001$ \\
\hline Mechanical prosthesis & $57(13.8 \%)$ & $109(42.1 \%)$ & $>.001$ \\
\hline Homograft & $7(1.7 \%)$ & $5(1.9 \%)$ & $>.99$ \\
\hline Aortic crossclamp time (min, mean \pm SEM) & $57.8 \pm 0.3$ & $61.6 \pm 0.5$ & $>.001$ \\
\hline Cardiopulmonary bypass time (min, mean \pm SEM) & $84.7 \pm 0.6$ & $89.5 \pm 0.8$ & $>.001$ \\
\hline
\end{tabular}

increases the risk of mortality and recurrent paravalvular leak. ${ }^{6,8}$

The use of pledgeted sutures has been suggested to decrease the risk of paravalvular leak after AVR. ${ }^{4}$ In their review of 807 patients undergoing mechanical heart valve replacement with 6 different suture techniques, Englberger and colleagues ${ }^{4}$ documented a $5.8 \%$ incidence of major paravalvular leak among patients undergoing mitral, aortic, and double valve replacements with a nonpledgeted suture technique. Moreover, on multivariate modeling, this group demonstrated that a nonpledgeted suture technique was an independent risk factor for major paravalvular leak events. Similarly, another study demonstrated that the use of small monofilament suture in a continuous fashion increased the risk of paravalvular leak relative to pledgeted sutures placed in a horizontal mattress fashion during mitral valve replace- ment. ${ }^{9}$ In that study, a nonpledgeted, horizontal mattress suture technique was not evaluated. Thus the effect of using nonpledgeted, horizontal mattress sutures on the incidence of major paravalvular leak exclusively after AVR has not been well defined.

In this study, major paravalvular leaks after isolated AVR were rare $(<1.5 \%)$, despite the use of a nonpledgeted suture technique, and the incidence of major paravalvular leak among patients undergoing primary, isolated nonpledgeted AVR was less than $1 \%$. With a mean follow-up of 82 months, we found that major paravalvular leak was detected at a mean of 51 months by routine transthoracic echocardiography. These results demonstrate the efficacious use of nonpledgeted sutures to secure a prosthetic valve to the aortic valve annulus without any increased risk of paravalvular leak relative to the use of a pledgeted suture technique. 
TABLE 3. Postoperative outcomes for patients undergoing isolated aortic valve replacement with a nonpledgeted versus pledgeted suture technique $(\mathbf{n}=\mathbf{8 0 2})$

\begin{tabular}{|c|c|c|c|}
\hline Variable & Nonpledgeted $(\mathrm{n}=511)$ & Pledgeted $(n=291)$ & $P$ value \\
\hline Sepsis (no.) & $8(1.6 \%)$ & $5(1.7 \%)$ & $>.99$ \\
\hline Stroke (no.) & $12(2.3 \%)$ & $6(2.1 \%)$ & $>.99$ \\
\hline Perioperative myocardial infarction (no.) & $2(0.4 \%)$ & $0(0.0 \%)$ & .54 \\
\hline Reoperation for bleeding or tamponade (no.) & $13(2.5 \%)$ & $7(2.4 \%)$ & $>.99$ \\
\hline Atrial fibrillation (no.) & $119(23.3 \%)$ & $82(28.8 \%)$ & .13 \\
\hline Heart block (no.) & $11(2.2 \%)$ & $8(2.7 \%)$ & .63 \\
\hline Cardiac arrest (no.) & $7(1.4 \%)$ & $7(2.4 \%)$ & .28 \\
\hline Gastrointestinal event (no.) & $7(1.4 \%)$ & $7(2.4 \%)$ & .28 \\
\hline Pneumonia (no.) & $19(3.7 \%)$ & $8(2.7 \%)$ & .55 \\
\hline Prolonged ventilation (no.) & $39(7.6 \%)$ & $13(4.5 \%)$ & $>.99$ \\
\hline Renal failure (no.) & $20(3.9 \%)$ & $18(6.2 \%)$ & .17 \\
\hline Hemodialysis (new onset, no.) & $6(1.2 \%)$ & $0(0.0 \%)$ & .09 \\
\hline \multicolumn{4}{|l|}{ Hospital stay $(\mathrm{d}$, mean $\pm \mathrm{SEM})$} \\
\hline Total & $8.6 \pm 0.4$ & $10.2 \pm 1.0$ & .11 \\
\hline Postoperative & $7.3 \pm 0.4$ & $7.4 \pm 0.8$ & .96 \\
\hline Paravalvular leak (no.) & $4(0.8 \%)$ & $4(1.4 \%)$ & .47 \\
\hline Reoperation for paravalvular leak (no.) & $1(0.2 \%)$ & $4(1.4 \%)$ & .06 \\
\hline Operative mortality (no.) & $13(2.5 \%)$ & $9(3.1 \%)$ & .66 \\
\hline
\end{tabular}

Further, the subsequent rate of reoperation for paravalvular leak was extremely low, with only 1 patient $(0.2 \%)$ undergoing reoperation after isolated nonpledgeted AVR. In our experience, a nonpledgeted suture technique does not increase the risk of reoperation after primary AVR.

Important patient characteristics among our study groups warrant further discussion. The incidences of preoperative infective endocarditis were similar in the groups and did not affect the major paravalvular leak rate after either nonpledgeted or pledgeted AVR. Patients undergoing AVR with pledgets had a higher proportion of mechanical valve implantation, whereas those undergoing nonpledgeted

TABLE 4. Patient demographic characteristics, preoperative risk factors, and operative features for patients incurring major paravalvular leaks after isolated, nonpledgeted or pledgeted aortic valve replacement $(n=8)$

\begin{tabular}{lcc}
\hline \multicolumn{1}{c}{ Variable } & $\begin{array}{c}\text { Nonpledgeted } \\
(\mathbf{n = 4 )}\end{array}$ & $\begin{array}{c}\text { Pledgeted } \\
(\mathbf{n}=\mathbf{4})\end{array}$ \\
\hline $\begin{array}{l}\text { Demographic data } \\
\text { Age (y, mean } \pm \text { SEM) }\end{array}$ & $67.3 \pm 5.2$ & $65.3 \pm 5.6$ \\
Sex (no. female) & $1(25.0 \%)$ & $1(25.0 \%)$ \\
Preoperative risk factors & & \\
$\quad$ Infective endocarditis (no.) & $0(0.0 \%)$ & $0(0.0 \%)$ \\
Aortic insufficiency (no.) & $1(25.0 \%)$ & $1(25.0 \%)$ \\
Aortic stenosis (no.) & $3(75.0 \%)$ & $3(75.0 \%)$ \\
Operative features & & \\
Bioprosthesis (no.) & $3(75.0 \%)$ & $1(25.0 \%)$ \\
$\quad \begin{array}{l}\text { Mechanical prosthesis (no.) } \\
\text { Aortic crossclamp time } \\
\quad(\text { min, mean } \pm \text { SEM) }\end{array}$ & $1(25.0 \%)$ & $3(75.0 \%)$ \\
Cardiopulmonary bypass time & $63.0 \pm 10.0$ & $64.0 \pm 9.0$ \\
$\quad($ min, mean \pm SEM) & $82.2 \pm 4.6$ & $83.2 \pm 3.6$ \\
\hline
\end{tabular}

AVR more commonly received bioprosthetic valves; however, these factors did not affect paravalvular leak rates. Further, observed aortic crossclamp and cardiopulmonary bypass times were statistically shorter among patients undergoing nonpledgeted AVR.

This study has several limitations. The retrospective design of this study introduces inherent bias. We were also

TABLE 5. Postoperative outcomes for patients undergoing primary, isolated aortic valve replacement with a nonpledgeted versus pledgeted suture technique $(n=671)$

\begin{tabular}{lrrr}
\hline \multicolumn{1}{c}{ Variable } & $\begin{array}{r}\text { Nonpledgeted } \\
(\mathbf{n = 4 1 2})\end{array}$ & $\begin{array}{c}\text { Pledgeted } \\
(\mathbf{n = 2 5 9 )}\end{array}$ & $\begin{array}{c}\boldsymbol{P} \\
\text { value }\end{array}$ \\
\hline Sepsis (no.) & $7(1.7 \%)$ & $5(1.9 \%)$ & $>.99$ \\
Stroke (no.) & $10(2.4 \%)$ & $5(1.9 \%)$ & .79 \\
Perioperative myocardial & $2(0.5 \%)$ & $0(0.0 \%)$ & .53 \\
$\quad$ infarction (no.) & & & \\
Reoperation for bleeding or & $12(2.9 \%)$ & $7(2.7 \%)$ & $>.99$ \\
$\quad$ tamponade (no.) & & & \\
Atrial fibrillation (no.) & $98(23.8 \%)$ & $76(29.3 \%)$ & .12 \\
Heart block (no.) & $8(1.9 \%)$ & $6(2.3 \%)$ & .79 \\
Cardiac arrest (no.) & $3(0.7 \%)$ & $4(1.5 \%)$ & .44 \\
Gastrointestinal event (no.) & $5(1.2 \%)$ & $6(2.3 \%)$ & .35 \\
Pneumonia (no.) & $13(3.2 \%)$ & $7(2.7 \%)$ & .82 \\
Prolonged ventilation (no.) & $26(6.3 \%)$ & $13(5.0 \%)$ & .61 \\
Renal failure (no.) & $14(3.4 \%)$ & $16(6.2 \%)$ & .12 \\
Hemodialysis (new onset, no.) & $4(1.0 \%)$ & $0(0.0 \%)$ & .30 \\
Hospital stay (d, mean \pm SEM) & & & \\
$\quad$ Total & $8.2 \pm 0.4$ & $10.5 \pm 1.1$ & .02 \\
$\quad$ Postoperative & $7.0 \pm 0.4$ & $7.5 \pm 0.9$ & .58 \\
Paravalvular leak (no.) & $2(0.5 \%)$ & $3(1.2 \%)$ & .38 \\
Reoperation for paravalvular & $0(0.0 \%)$ & $3(1.2 \%)$ & .06 \\
$\quad$ leak (no.) & & & \\
Operative mortality (no.) & $8(1.9 \%)$ & $6(2.3 \%)$ & .79 \\
\hline
\end{tabular}


limited by the definitions and constraints of the variables included in the STS database. The low incidence of major paravalvular leak and low operative mortality after AVR operations limited our ability to apply meaningful statistical modeling for either adjusted outcomes or survival analysis. The confounding effects of numerous operating surgeons, as well as changes in both cardiac anesthesia and postoperative care with time, are difficult to assess in data analysis. Finally, larger sample sizes might have increased the ability to detect small differences during group comparisons. Nevertheless, the equivalent outcomes in mortality, major paravalvular leak rate, and need for aortic valve reoperation between nonpledgeted and pledgeted AVRs, coupled with statistically shorter aortic crossclamp and cardiopulmonary bypass times in the nonpledgeted cohort, highlights the important clinical contribution of this study to the cardiac literature.

\section{CONCLUSIONS}

We conclude that a nonpledgeted suture technique offers an equivalent alternative to the traditional use of pledgets during open aortic valve replacement, with no increase in major paravalvular leak rate. Use of a nonpledgeted suture technique provides a time efficient and safe approach to aortic valve replacement operations.
We thank Curtis Klann and Judy Smith for their assistance with the STS database search and data collection.

\section{References}

1. Society of Thoracic Surgeons. Society of Thoracic Surgeons national cardiac surgical database. Chicago: Society of Thoracic Surgeons. 2008 [cited 2009]. Available at: http://www.sts.org/sections/stsnationaldatabase/.

2. Aronow WS, Kronzon I. Prevalence and severity of valvular aortic stenosis determined by Doppler echocardiography and its association with echocardiographic and electrocardiographic left ventricular hypertrophy and physical signs of aortic stenosis in elderly patients. Am J Cardiol. 1991;67:776-7.

3. Jaussaud N, Gariboldi V, Giorgi R, Grisoli D, Chalvignac V, Thuny F, et al. Risk of reoperation for aortic bioprosthesis dysfunction. J Heart Valve Dis. 2009;18: 256-61.

4. Englberger L, Schaff HV, Jamieson WR, Kennard ED, Im KA, Holubkov R, et al. Importance of implant technique on risk of major paravalvular leak (PVL) after St. Jude mechanical heart valve replacement: a report from the Artificial Valve Endocarditis Reduction Trial (AVERT). Eur J Cardiothorac Surg. 2005;28:838-43.

5. Rallidis LS, Moyssakis IE, Ikonomidis I, Nihoyannopoulos P. Natural history of early aortic paraprosthetic regurgitation: a five-year follow-up. Am Heart $J$. 1999;138:351-7.

6. Jindani A, Neville EM, Venn G, Williams BT. Paraprosthetic leak: a complication of cardiac valve replacement. J Cardiovasc Surg (Torino). 1991;32:503-8.

7. Miller DL, Morris JJ, Schaff HV, Mullany CJ, Nishimura RA, Orszulak TA. Reoperation for aortic valve periprosthetic leakage: identification of patients at risk and results of operation. J Heart Valve Dis. 1995;4:160-5.

8. Jones JM, O'Kane H, Gladstone DJ, Sarsam MA, Campalani G, MacGowan SW, et al. Repeat heart valve surgery: risk factors for operative mortality. J Thorac Cardiovasc Surg. 2001;122:913-8.

9. Dhasmana JP, Blackstone EH, Kirklin JW, Kouchoukos NT. Factors associated with periprosthetic leakage following primary mitral valve replacement: with special consideration of the suture technique. Ann Thorac Surg. 1983;35:170-8. 